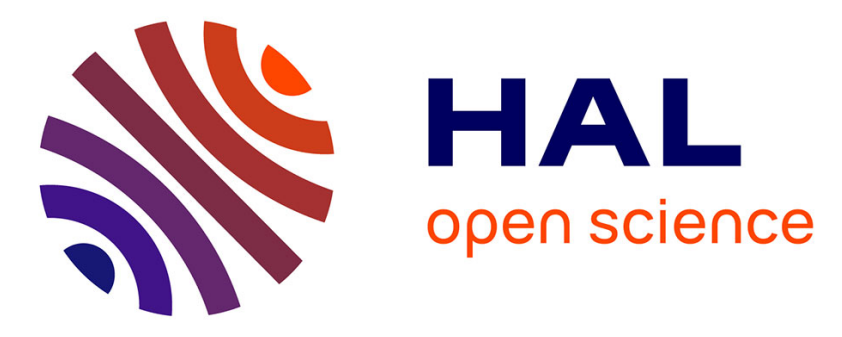

\title{
Inelastic light scattering by long narrow gold nanocrystals: when size, shape, crystallinity and assembly matter
}

Hervé Portalès, Nicolas Goubet, Sandra Casale, Xiang Zhen Xu, Mostapha Ariane, Alain Mermet, Jeremie Margueritat, Lucien Saviot

\section{To cite this version:}

Hervé Portalès, Nicolas Goubet, Sandra Casale, Xiang Zhen Xu, Mostapha Ariane, et al.. Inelastic light scattering by long narrow gold nanocrystals: when size, shape, crystallinity and assembly matter. ACS Nano, 2020, 14 (4), pp.4395-4404. 10.1021/acsnano.9b09993 . hal-02566470

\author{
HAL Id: hal-02566470 \\ https://hal.science/hal-02566470
}

Submitted on 7 May 2020

HAL is a multi-disciplinary open access archive for the deposit and dissemination of scientific research documents, whether they are published or not. The documents may come from teaching and research institutions in France or abroad, or from public or private research centers.
L'archive ouverte pluridisciplinaire HAL, est destinée au dépôt et à la diffusion de documents scientifiques de niveau recherche, publiés ou non, émanant des établissements d'enseignement et de recherche français ou étrangers, des laboratoires publics ou privés. 


\title{
Inelastic Light Scattering by Long Narrow Gold Nanocrystals: When Size, Shape, Crystallinity and Assembly Matter
}

\author{
Hervé Portalès, ${ }^{* \dagger}$ Nicolas Goubet, ${ }^{*, \dagger}$ Sandra Casale, ${ }^{\ddagger}$ Xiang Zhen Xu,

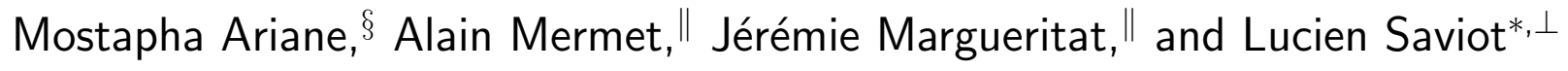 \\ † Sorbonne Université, CNRS, MONARIS, UMR 8233, 4 place Jussieu, 75005 Paris, \\ France \\ $\ddagger$ Sorbonne Université, CNRS, LRS, UMR 7197, 4 Place Jussieu, 75005 Paris, France \\ ฯ LPEM, ESPCI Paris, PSL University, CNRS, 75005 Paris, France; \\ Sorbonne Université, CNRS, LPEM, UMR 8213, 4 place Jussieu, 75005 Paris, France \\ $\S$ SAYENS-SATT Grand Est, 64A rue Sully, 21000 Dijon, France \\ || Institut Lumière Matière, UMR 5306, Université Lyon 1-CNRS, Université de Lyon, \\ 69622 Villeurbanne, France \\ $\perp$ Laboratoire Interdisciplinaire Carnot de Bourgogne, UMR 6303 CNRS-Université \\ Bourgogne Franche-Comté, 9 Avenue Alain Savary, BP 47 870, 21078 Dijon Cedex, France \\ E-mail: herve.portales@sorbonne-universite.fr; nicolas.goubet@sorbonne-universite.fr; \\ lucien.saviot@u-bourgogne.fr
}

\begin{abstract}
We report the synthesis of long narrow gold nanocrystals and the study of their vibrational dynamics using inelastic light scattering measurements. Rich experimental spectra
\end{abstract}


are obtained for monodomain gold nanorods and pentagonal twinned bipyramids. Their assignment involves diameter-dependent non-totally symmetric vibrations which are modeled in the framework of continuum elasticity by taking into account simultaneously the size, shape and crystallinity of the nanocrystals. Light scattering by vibrations with angular momenta larger than 2 is reported. It is shown to increase with the ratio of the nanocrystals diameter to the interparticle separation. It originates from the plasmonic coupling due to the self-assembly of the nanocrystals after deposition.

\section{Keywords}

gold nanocrystals, nanorods, nanobipyramids, plasmonic coupling, Raman scattering, eigenvibrations, anisotropy

Gold nanocrystals have been studied extensively in the last few decades as they are promising candidates for a variety of applications in different fields including sensing, catalysis and biomedicine. 13 This is due to the tunability of their optical properties, plasmonic properties, when varying their composition, size, shape ${ }^{4}$ and environment. During the last twenty years the mechanical properties of gold nanocrystals, their vibrational properties, have been shown to also strongly depend on these parameters and to provide valuable information about the nanostructures themselves (size, shape, crystallinity) and their environment. 521 This correlated dependency of the optical and mechanical properties make gold nanoparticles the simplest opto-mechanical transducers where they act both as source and detector. ${ }^{22}$ Such optical and mechanical properties can be tuned by controlling their structural properties and environment. Moreover, recent works in this domain have shed a new light on the coupling between the optical and mechanical properties by showing that the inelastic light scattering process is governed by the variations of the electric field inside the nanoparticles even for very small nanospheres. ${ }^{9123}$ By providing a way to probe these variations in particular under resonant excitation, inelastic light scattering may prove as valuable 
for the study of surface plasmons in metallic nanoparticles as resonant Raman scattering has been for the study of the electronic properties of other systems. $\underline{24} \underline{26}$

Analytical models exist to calculate the inelastic light scattering cross-sections ${ }^{9223}$ of metallic nanoparticles. They are restricted to systems having the spherical symmetry. They were developed thanks to numerous experimental results reported over the years for gold $\underline{5[11}$ and also other metallic nanoparticles. ${ }^{27 / 28}$ One of the goals of the present paper is to provide measurements for non-spherical nanocrystals to help develop models for non-spherical shapes. Indeed, experimental inelastic light scattering measurements reported in the literature are scarce. Various authors have investigated the vibrational dynamics of long, narrow gold nanoparticles but mostly using time-resolved transient absorption with femtosecond pumpprobe measurements. $\frac{12[14] 16 / 18}{16}$ This method is extremely powerful and even more so when it is used to investigate single particles. $\frac{29}{29}$ However, it also suffers from very restrictive selection rules. The observable vibrations are the totally symmetric ones because these are the only ones showing a variation of the volume of the particles during its oscillation. ${ }^{30}$ Inelastic light scattering (Raman/Brillouin) has less restrictive selection rules which make it possible to observe additional vibrations. In addition, the Raman selection rules are relaxed for large nanoparticles ${ }^{31}$ and when interparticle plasmonic coupling exists. $\frac{19}{19}$ The very low crosssections of the inelastic light scattering process prevent single-particle measurements in most cases. Still, ensemble measurements combined with narrow size dispersion have been used successfully in the past to investigate spherical nanoparticles and also in the case of transient absorption measurements when single-particle measurements remain difficult. 32

In this work, we prepared narrow size dispersion elongated gold nanocrystals which are suitable for ensemble measurements without introducing a dramatic inhomogeneous broadening. We controlled their morphology and measured their size with electron microscopy and optical absorption. Then we measured their inelastic light scattering spectra. Previous works have generally focused on totally symmetric vibrations (the so-called extensional and breathing modes $)^{33}$ even in the case of semiconducting nanoparticles. ${ }^{34}$ In that case, 
crystallinity only results in minor deviations compared to the case of elastically isotropic nanoparticles. For example, a coupling of the breathing vibration with $\mathrm{m}=5$ vibrations for pentagonal structures has been reported ${ }^{35}$ together with the necessity to take into account the right sound velocity corresponding to the lattice direction along the long axis for the extensional mode. ${ }^{36 \mid 37}$ However, for nanorods (NRs) additional vibrations can also significantly scatter light, $\frac{38}{3}$ some of which are very sensitive to the crystallinity of the nanocrystals. .39 Accordingly, we consider in this work only nanocrystals with a controlled lattice structure, i.e., monodomain NRs and twinned pentagonal nanobipyramids (NBP). In addition, a tandem Fabry-Pérot interferometer is used to measure the spectra. It enables measurements in a lower frequency range than conventional low-frequency Raman spectrometers (down to about $1 \mathrm{GHz}$ ). This enables to study larger nanoparticles for which deviations from the Raman scattering rules are expected.

\section{Results and Discussion}

\section{Morphology}

Colloidal solutions of Au NRs and NBPs were synthesized using the protocols described in the Methods section. In the various colloidal solutions, the size distribution of the NRs remains as narrow as a few percents. Such a low polydispersity allows for getting NR selfassembly after depositing them onto a Si substrate, as observed by high-resolution scanning electron microscopy (HRSEM) in Figure 1A. When looking at the high resolution transmission electron microscopy (HRTEM) image of one single NR in Figure 1B, the apparent profile is well described by a cylinder with half-sphere ends (Figure 1C). A similar profile is observed for most of the synthesized NRs. In the following we model them as half-sphere ended cylinders of total length $L$ and diameter $d$. All of them are single domain with their long axis along [001].

Figure 1D shows the length versus diameter distributions of the six samples. The aspect 

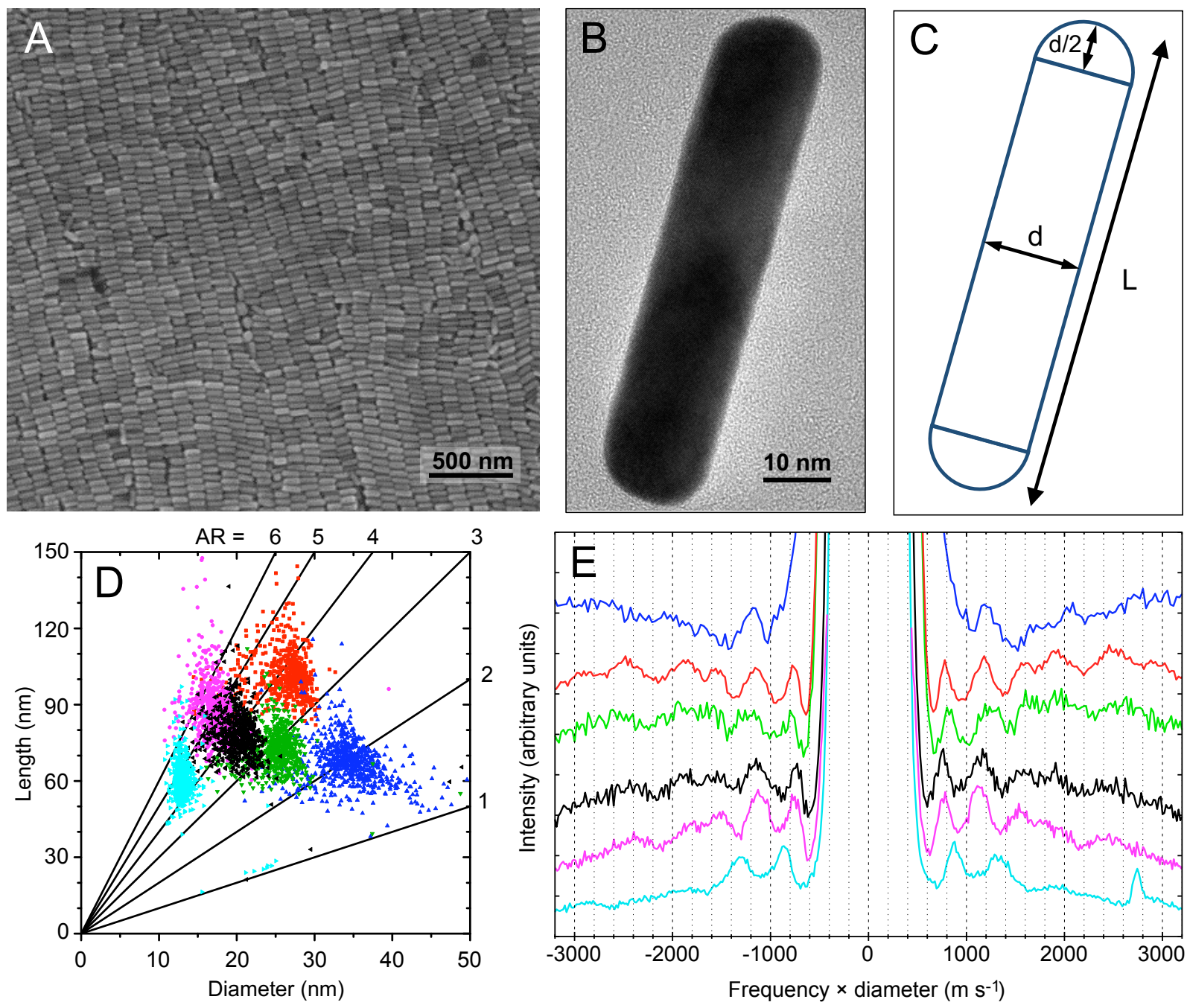

Figure 1: (A) HRSEM image of NR\#5 nanocrystals self-assembled on a Si wafer. (B) HRTEM image showing one typical single domain Au NR. (C) Schematics depicting the relevant NR dimensions. As a first approximation, each NR can be modeled by a cylinder of diameter $d$ ended with two half-spheres, with a total length $L$. (D) Length versus diameter distributions of $\mathrm{Au}$ NRs of samples NR\#1 (light blue), NR\#2 (pink), NR\#3 (black), NR\#4 (green), NR\#5 (red) and NR\#6 (blue). Continuous straight lines are plotted as a guide to the eyes to materialize specified values of the aspect ratio. (E) Stokes and anti-Stokes low-frequency Raman scattering spectra of Au NRs with different sizes and aspect ratios as a function of the frequency shift multiplied by the average NR diameter. The spectra of samples NR\#i $(i=1-6)$ are plotted from bottom to top with the same colors as in D. They are independently scaled and vertically shifted for clarity. 
ratio of the NRs (AR), defined as the average ratio of the length to the diameter $\langle L / d\rangle$, changes significantly from one sample to another. This variation reflects our ability to independently increase the diameter and length of the NRs. In other words, no correlation is found between these two dimensions in the synthesized NRs. Aspect ratio, average diameter and length are given in Table 1 for the different NR\#i samples listed in increasing order of the average NR diameter. The diameter varies almost by a factor 3 from sample NR\#1 to sample NR\#6. The average length only varies by a factor 1.7 between samples NR\#1 and $\mathrm{NR} \# 5$. The aspect ratio ranges from $\mathrm{AR} \simeq 2$ in sample $\mathrm{NR} \# 6$ to $\mathrm{AR} \simeq 5$ in sample $\mathrm{NR} \# 2$. More details about the diameter and length distributions of the NRs for all samples are given in Figures S1-S6 in the SI.

Table 1: (A) Average diameters, lengths and aspect ratios for samples NR\#i $(i=1-6)$ and NBP\#i $(\mathrm{i}=1-3)$ with the corresponding standard deviation in parentheses. The standard deviation for $t$ was taken as $0.3 \times t$ to account for the lower reliably of determining this length from the TEM images.

\begin{tabular}{rrrrr} 
NR & $d(\mathrm{~nm})$ & $L(\mathrm{~nm})$ & $L / d$ & \\
$\# 1$ & $13.1(1.4)$ & $60.3(7.6)$ & $4.65(0.70)$ & \\
$\# 2$ & $17.3(2.7)$ & $87.3(11.8)$ & $5.14(1.11)$ & \\
$\# 3$ & $20.1(2.6)$ & $78.3(9.7)$ & $3.96(0.70)$ & \\
$\# 4$ & $25.3(2.7)$ & $72.1(7.5)$ & $2.88(0.41)$ & \\
$\# 5$ & $26.1(3.1)$ & $101.7(9.6)$ & $3.95(0.64)$ & \\
$\# 6$ & $34.4(3.9)$ & $67.3(8.8)$ & $1.99(0.37)$ & \\
\hline NBP & $w(\mathrm{~nm})$ & $L(\mathrm{~nm})$ & $L / w$ & $t(\mathrm{~nm})$ \\
$\# 1$ & $23.5(4.3)$ & $56.5(9.6)$ & $2.50(0.77)$ & $13.8(4.1)$ \\
$\# 2$ & $26.1(2.7)$ & $66.0(9.6)$ & $2.53(0.23)$ & $14.0(4.2)$ \\
$\# 3$ & $51.5(3.8)$ & $151.0(8.6)$ & $2.95(0.24)$ & $15.4(4.6)$
\end{tabular}

Similarly, HRSEM and HRTEM images were obtained for NBPs. They are presented in Figures $2 \mathrm{~A}$ and $2 \mathrm{~B}$ together with a schematic representation (Figure $2 \mathrm{C}$ ). Our observation matches the morphology reported in the literature. ${ }^{40}$ The average length, width at the center and aspect ratio are also given in Table 1. 

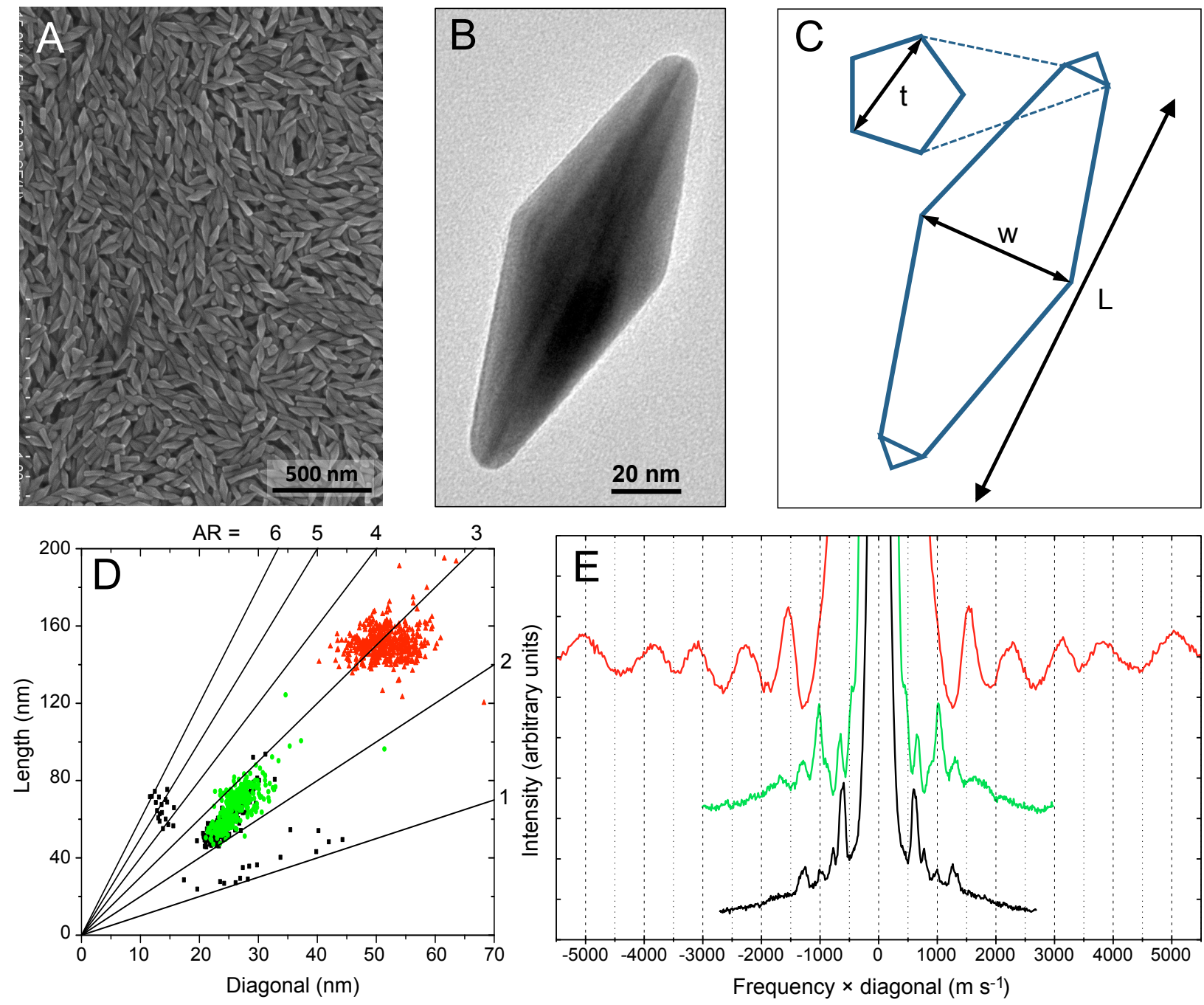

Figure 2: (A) HRSEM image of NBP\#3 nanocrystals self-assembled on a Si wafer. (B) HRTEM image showing one typical pentagonal Au NBP. (C) Schematics depicting the relevant NBP dimensions. As a first approximation, each NBP can be modeled by a pentagonal biprysm of diagonal at the center $w$ ended with two pyramids of diagonal $t$, with a total length $L$. (D) Length versus diagonal distributions of Au NBPs of samples NBP\#1 (black), NBP\#2 (green) and NBP\#3 (red). (E) Stokes and anti-Stokes low-frequency Raman scattering spectra of $\mathrm{Au}$ NBPs with different sizes and aspect ratios as a function of the frequency shift multiplied by the average NBP diagonal at the center. The spectra of samples NBP\#i (i=1-3) are plotted from bottom to top with the same colors as in $\mathrm{D}$. The spectra are independently scaled and vertically shifted for clarity. 


\section{Raman spectra}

Raman measurements were performed on NRs deposited onto a Si wafer after evaporation of the colloidal solution. No control of the orientation of the NRs is achieved, meaning that these latter are randomly oriented with respect to the polarization of the incident and scattered light. The low-frequency Raman spectra of samples NR\#i are shown in Figure S8. Each spectrum exhibits several bands in the $30-150 \mathrm{GHz}$ frequency range. The peaks do not overlap significantly which demonstrates that the control of the size distributions of the NRs during the synthesis was good enough for this study. The bands shift towards higher frequencies for decreasing diameters. To highlight the dependence of the low-frequency Raman spectra on the NR diameter, we plotted them in Figure $1 \mathrm{E}$ as a function of $\nu d$, the product of the frequency $\nu$ by the diameter $d$. This scaling comes from the continuum elasticity approximation used in the calculations. The frequencies scale as the inverse of the dimensions provided the aspect ratio is kept constant. In a previous work, it was shown that the eigenmodes of NRs are governed mainly by either the length or the diameter. ${ }^{[39}$ We take advantage of this scaling law in the following by considering the product of the frequency by the length and by the diameter of the NRs. They are expressed in $\mathrm{m} / \mathrm{s}$ when multiplying frequencies in $\mathrm{GHz}$ by lengths in $\mathrm{nm}$. Two bands at about $\nu d=800$ and $1200 \mathrm{~m} / \mathrm{s}$ dominate the spectra. These bands hardly move from one spectrum to another, thus indicating that they originate from two vibrational modes with frequencies scaling as $1 / d$. In spite of a weaker signal/noise ratio of the spectra at higher frequency, one discerns other bands whose $\nu d$ products remain also almost unchanged. At variance with the extensional modes detected by transient absorption measurements and whose frequency varies as $1 / L$, the frequency of most of the vibrational modes detected here scales approximately as $1 / d$. Therefore, the

multiple features observed in the Raman spectra are probably due to radial modes of the NRs. Their assignment will be discussed in the following.

Three lengths are required to describe the shape of NBPs: the total length $L$ and the width at the center $w$ and at both ends $t$ (see Figure $2 \mathrm{C}$ ). For pentagonal cross-sections, 
the lateral dimension is chosen as the diagonal of the pentagon because this is the width which we expect to observe in HRTEM images when NBPs rest on one of their faces. We use the same representation as for the NRs and scale the frequencies by multiplying them by the diagonal of the bipyramids at the center $(\nu w)$. This enables to easily compare peaks for samples having different sizes. The Raman spectra of the NBP\#i samples are presented in Figure 2 $\mathrm{E}$. We note that they are as rich as in the case of NRs because they all contain 5 peaks or more. The lowest frequency peaks for NBP\#1 and NBP\#2 are at lower value of $\nu w$ and may therefore correspond to modes related to the length of the NBPs. The case of NBP\#3 is similar to the case of the NRs before with the peaks appearing at larger $\nu w$ corresponding most probably to vibrations confined along the lateral dimension.

A $647 \mathrm{~nm}$ laser excitation was used to measure the inelastic light scattering spectra. According to the UV-visible absorption spectra (Figures S14 and S15), the excitation is strongly resonant for the samples with the smallest aspect ratio. It falls in between the longitudinal and transverse surface plasmon resonances for the samples with the largest aspect ratio. However, these spectra were measured for the colloidal solution. The selfarrangement of the nanocrystals after deposition most probably alters this simple picture. We chose the $647 \mathrm{~nm}$ laser excitation in the present work to favor a resonant excitation and facilitates the measurement of the spectra. Future works will have to look more closely at possible resonance effects which may enhance the scattering of some nanocrystals depending on their size or assembly.

The assignment of the experimental peaks is achieved by comparing their frequencies with calculated ones. There is never a perfect match in practice due to the size distributions and the approximations of the calculation method. Comparing frequencies works well for the lowest frequency vibrations where the different modes are well separated. The increase of the vibrational density of states with frequency renders this approach much less effective at higher frequencies. In the present case, the lowest frequency modes are the extensional ones. The features observed for the NRs are mainly diameter-dependent and are therefore 
not the lowest frequency modes. To assign the peaks, we use selection rules when possible to restrict the number of modes to consider.

Recent works have also shown that the plasmonic coupling between neighbor nanoparticles can break the usual Raman selection rules ${ }^{19}$ resulting in a larger number of peaks in the spectra. In that case, the additional experimental features observed for spheres have been successfully assigned to the fundamental spheroidal vibrations with $\ell=3,4, \ldots$ Such a coupling is possible in our samples because the nanocrystals tend to self-assemble as can be seen in the HRSEM images (Figures $1 \mathrm{~A}$ and $2 \mathrm{~A}$ ). Therefore, a similar assignment can be expected. To test this hypothesis, we focus in the following on describing how these spheroidal vibrations evolve when changing the shape from spheres to NRs and NBPs. Then we try to assign the spectral features with these vibrations. The same spheroidal vibrations with $\ell=3,4, \ldots$ manifest also when the size of the nanoparticles is not small compared to the wavelength of light. ${ }^{31}$ However, we do not anticipate that the same size effect manifests in our case. Indeed, vibrations with $\ell \neq 2$ are not observed for gold nanospheres ${ }^{19}$ having a diameter of about $100 \mathrm{~nm}$ which is close to the length of our elongated nanocrystals.

\section{Monodomain NRs}

We start from the well-known case of the isotropic nanosphere of diameter $d$, then change the shape to an isotropic circular cylinder and then to a cubic circular cylinder as an approximation for a monodomain NR.

The diameter-dependent modes of the cylinder corresponding to the fundamental spheroidal modes of the sphere with angular momentum $\ell>0$ are the ones with $m=\ell$ at the center of the Brillouin zone $(q=0)$. They are the lowest frequency $\mathrm{E}_{m \mathrm{u}}$ for odd $m$ or $\mathrm{E}_{m \mathrm{~g}}$ for even $m>0 . \underline{39}$ The corresponding surface deformation for a cross-section of the isotropic gold cylinder is plotted in Figure 3A (top). For these $q=0$ modes, the deformation is the same along the cylinder. The frequencies $\nu$ of these modes vary as the inverse diameter $1 / d$. The diameter-independent values $\nu d$ are given in Figure $3 \mathrm{~A}$ and plotted in Figure $3 \mathrm{~B}$ with 
squares as a function of $m$. For comparison, the spheroidal modes of the sphere are also plotted with circles as a function of $\ell$. The frequencies are smaller for the cylinder but the variations with $\ell$ or $m$ are very similar. Overtones of the spheroidal vibrations of the sphere are also interesting in this context. However, their scattering cross-sections are substantial only if they change the shape of the surface significantly. For spherical nanoparticles, the

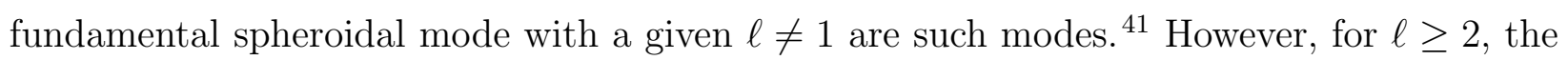
next 2 or 3 overtones keep the shape of the nanosphere almost unchanged and their scattering cross-sections are small. $\frac{9123}{2}$ We checked that a similar behavior still holds for cylinders. For this reason, we will only consider the fundamental modes in the following.

The spheroidal-like modes of isotropic cylinders with even $m=2 i>0$ have the $\mathrm{E}_{(2 i) \mathrm{g}}$ irreducible representation. For single-crystal cylinders made of a cubic material, they split into $\mathrm{B}_{1 \mathrm{~g}}$ and $\mathrm{B}_{2 \mathrm{~g}}$ for odd $i$ and $\mathrm{A}_{1 \mathrm{~g}}+\mathrm{A}_{2 \mathrm{~g}}$ for even $i$. For odd $m=2 i+1$, the isotropic spheroidal-like modes have the $\mathrm{E}_{(2 i+1) \mathrm{u}}$ irreducible representation. They do not split and transform into $\mathrm{E}_{\mathrm{u}} \cdot{ }^{39}$ For $m=0$, the breathing mode of the sphere transforms into the radial breathing mode of the cylinder and the irreducible representation remains unchanged $\left(\mathrm{A}_{1 \mathrm{~g}}\right)$. The first few modes shapes and frequencies $(m=0, \ldots 6)$ are illustrated in Figure $3 \mathrm{~A}$ (middle). The deformations for the isotropic and cubic vibrations are almost identical for $m=2$. They differ for modes at higher frequencies due to mixing with other modes having the same cubic irreducible representations and close frequencies. Such near frequency modes are more frequent at higher frequency due to the increasing vibrational density of states. Only the cubic vibrations being the most similar to the isotropic ones are considered here. As expected from previous works, $\frac{11}{1}$ the significant splitting of the $\mathrm{E}_{2 \mathrm{~g}}$ mode into $\mathrm{B}_{1 \mathrm{~g}}$ and $\mathrm{B}_{2 \mathrm{~g}}$ is a clear marker of the cubic lattice structure of gold in circular cylinders. The frequency shifts for the other modes are also significant. This demonstrates that taking into account this anisotropic structure is required.

Figure $3 \mathrm{C}$ was designed to help assign the peaks observed in the experimental spectra. The axes correspond to $\nu d$ and $\nu L$ to help distinguish between diameter and length dependent 


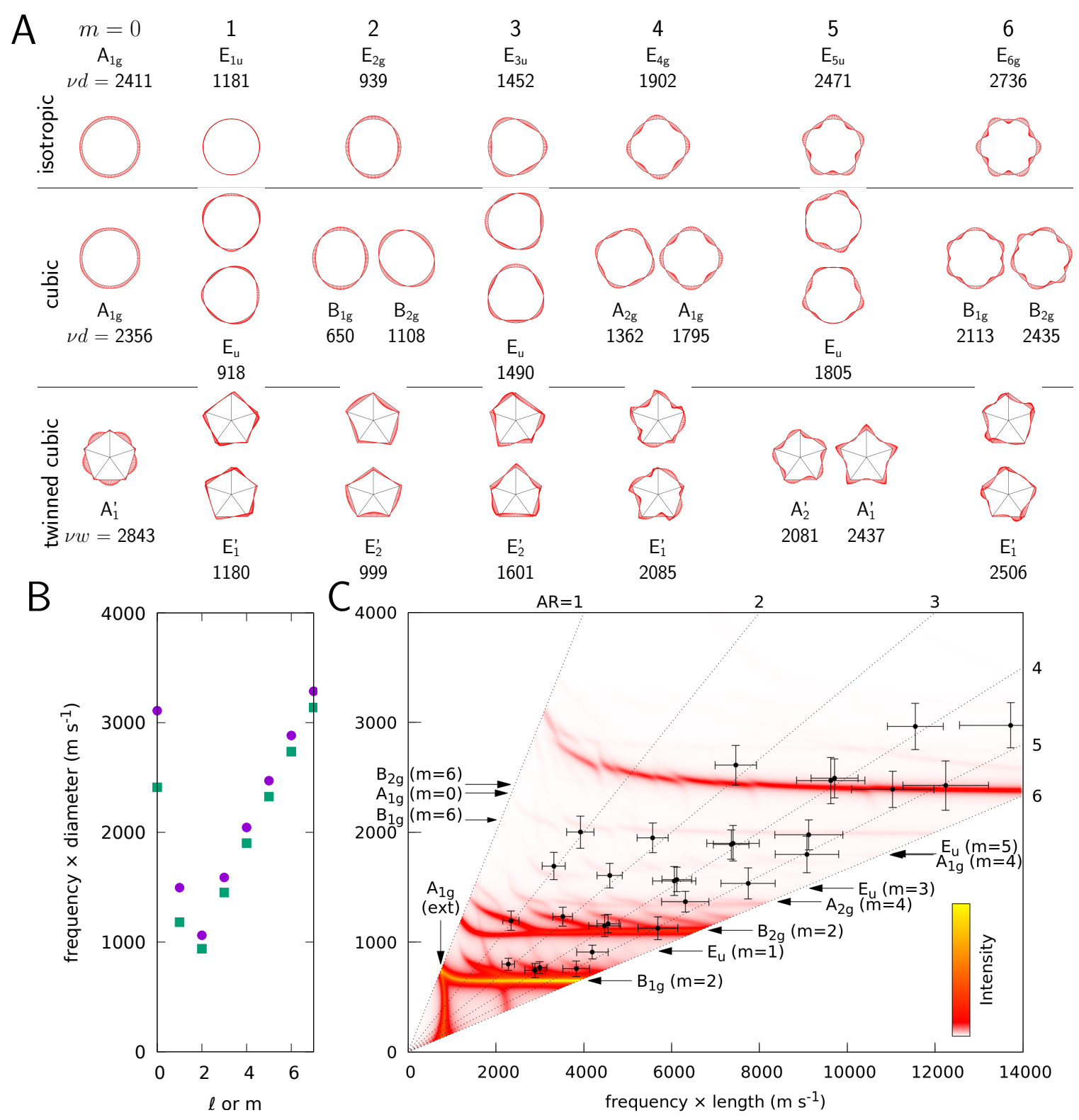

Figure 3: (A) Surface deformation of isotropic gold cylinders deformed by the $q=0$ radial vibrations with $m=0, \ldots, 6$ (top). The second degenerate isotropic mode is obtained by rotating by $\frac{\pi}{2 m}$. The equivalent vibrations are also shown for cubic gold cylinders (middle) and for cubic twinned pentagonal gold cylinders (bottom). The irreducible representations and $\nu d$ (frequency $\times$ diameter in $\mathrm{m} / \mathrm{s}$ ) or $\nu w$ (frequency $\times$ diagonal of the pentagon in $\mathrm{m} / \mathrm{s}$ ) are indicated. (B) $\nu d$ for the fundamental spheroidal modes of an isotropic gold sphere (circles) and cylinder (square) as a function of the angular momentum $\ell$ or $m$. (C) Color map of the intensity calculated using the Brillouin mechanism as a function of $\nu L$ and $\nu d$ for monodomain circular NRs with half sphere ends which are made of cubic gold. Experimental frequencies are plotted with full circles and the errorbars correspond to the FWHM of the diameter and length distributions. The frequencies of the "spheroidal-like" $q=0$ diameterdependent modes of circular cylinders made of cubic gold are indicated with arrows together with their irreducible representations. 
modes. The intensities calculated according to the Brillouin mechanism ${ }^{39}$ are plotted as a $2 \mathrm{D}$ color map as a function of $\nu d$ and $\nu L$. It was obtained by calculating the intensities for NRs having varying aspect ratios. In such a plot, the eigenmodes governed mainly by the length of the NRs (such as the extensional modes) appear as vertical lines while the ones governed by the diameter (such as the breathing mode) appear as horizontal lines. The experimental peak positions for the different samples are plotted with circles and error bars. The circles are positioned using the average diameter and length of the NRs. The error bars are obtained by taking into account the full width at half maximum (FWHM) of the diameter and length distributions. The modes of interest of the cubic circular cylinder at $q=0$ are represented as horizontal arrows at the corresponding $\nu d$ and their irreducible representations are indicated. According to reference 39, the experimental $\nu d$ values should tend toward the calculated $\nu d$ values for $q=0$ modes as the aspect ratio $L / d$ increases.

The model used for the calculation of the color map predicts significant scattering by the extensional modes (vertical line at $\nu L \simeq 800 \mathrm{~m} / \mathrm{s}$ ), the "quadrupolar-like" vibrations (lowest frequency $\mathrm{B}_{1 \mathrm{~g}}$ and $\mathrm{B}_{2 \mathrm{~g}}$ at $\nu d \simeq 650$ and $\left.1100 \mathrm{~m} / \mathrm{s}\right)$ and the breathing mode $(\nu d \simeq$ $2350 \mathrm{~m} / \mathrm{s}$ ). The extensional modes lie at lower frequency and were not resolved in this work. Three peaks in the spectra correspond to the three other modes. The agreement is not very good for the $\mathrm{B}_{1 \mathrm{~g}}$ mode for which the calculations underestimate the frequencies. Peaks in the $1400<\nu d<2000 \mathrm{~m} / \mathrm{s}$ range are not predicted by this model. However, they are qualitatively well taken into account by considering modes coming from larger values of $\ell$ (or $m$ ) as discussed before. In particular, two branches consisting of peaks observed for most samples are close to the $\mathrm{E}_{\mathrm{u}}(m=3)$ and $\mathrm{A}_{1 \mathrm{~g}}(m=4)$ modes. We note that sample NR\#1 has a slightly different behavior. Its lowest frequency peaks match better with the $\mathrm{E}_{\mathrm{u}}(m=1)$ and $\mathrm{A}_{2 \mathrm{~g}}(m=4)$ modes. Assigning peaks to the $m=1$ vibration is unusual. Indeed, these vibrations do not significantly scatter light in the case of isotropic material because they do not change the shape of the surface. However, this is not true when the cubic lattice structure is present as can be seen in Figure 3A. Our experimental results do 
not allow us to explain the reason for the different behavior of sample NR\#1 at this point. It could be related to a different arrangement of the NRs in the region illuminated by the laser. Indeed, our assignment is based on identifying the vibrations which couple significantly with the surface plasmon resonance. However, the relative inelastic light scattering cross-sections of these modes are expected to depend significantly on the relative orientation of the NRs and the polarization of the laser but also on the position of the excitation wavelength with respect to the surface plasmon resonance.

The breaking of the Raman selection rules induced by plasmonic coupling and resulting in the scattering of light by vibrations with angular momentum larger than 2 was not observed in previous measurements on self-assembled nanoparticles. $\frac{11 / 42 / 43}{1}$ The reason for this apparent contradiction is the different coupling regime achieved in the present work. An efficient coupling for gold nanorods requires separation distances shorter than the nanorod diameter.44 A universal scaling behavior of the plasmonic coupling has been reported for dimers. $\stackrel{45}{4}$ We expect a similar law to exist for self-assembled nanoparticles, i.e., that the plasmonic coupling decreases quickly as a function of the ratio $s / D$ where $s$ is the separation distance between the nanoparticles and $D$ their size. $s / D \simeq \frac{1}{3}$ for samples prepared with pulsed laser deposition. $\frac{42}{42}$ The separation distance was about $1 \mathrm{~nm}$ leading to $s / d \simeq \frac{1}{5}$ for previous works on small NCs $(d \sim 5 \mathrm{~nm}) \stackrel{11 / 43}{ }$ In the present work, $s \simeq 2 \mathrm{~nm}$ leading to $s / d \simeq \frac{1}{10}$. This smaller value correspond to a stronger plasmonic coupling in agreement with the larger observed deviation from the usual Raman selection rules. The effect is even more pronounced if we consider the length of the $\operatorname{NRs}\left(s / L \simeq \frac{1}{50}\right)$. The deviation from the Raman selection rules as the size of the nanoparticles increases is also observed in our spectra. We can safely compare samples NR\#3 and NR\#5 because they have almost the same aspect ratio. The NRs of sample NR\#5 are about $30 \%$ larger than those of sample NR\#3. More Raman peaks are observed for NR\#5 than for NR\#3 in Figure 1 $\mathrm{E}$ which is again consistent with a stronger plasmonic coupling.

Finally, we note that the breaking down of the Raman selection rules which we report 
is quite dramatic. In the case of gold nanodimers, $\frac{19}{19}$ the intensities of the additional peaks assigned to vibrations with $\ell>2$ was about one order of magnitude lower than the one for $\ell=2$. In the present work, the intensity of the peaks are about the same (see Figure 1). We note that each NR has several neighbor NRs in our deposited samples instead of a single one in nanodimers. Therefore, the plasmonic coupling occurs over a larger fraction of the surface of the NRs which may explain at least in part the larger intensities that we observed. In addition, it is well-known that the electric field is intense and varies strongly over short distances near the tips of NRs. This can also explain the large intensities for $\ell>2$ vibrations when the excitation wavelength is near the longitudinal surface plasmon resonance.

\section{Pentagonal NBPs}

The $\mathrm{E}_{(2 \mathrm{i}+1) \mathrm{u}}$ and $\mathrm{E}_{(2 \mathrm{i}) \mathrm{g}}$ modes at $q=0$ of the isotropic circular cylinders split in pentagonal cylinders when the angular momentum $m=2 i(+1)$ is a multiple of 5 . The corresponding surface deformation and frequency $\times$ diagonal products are given in Figure 3 A (bottom)for cubic twinned pentagonal cylinders. We used the lattice orientations given in reference 40 , Contrarily to the case of monodomain cylinders detailed above, taking into account the cubic elasticity plays only a minor role for twinned pentagonal cylinders. In a simple approach, we can consider that vibrations of actual bipyramids are intermediate between those of spheres (for $L \sim w$ ) and pentagonal cylinders $(L \gg w)$. We checked this hypothesis for the lowest frequency $\mathrm{E}_{2}^{\prime}$ mode (quadrupolar-like) calculated using the finite element method and using the average dimensions given in Table 1. We obtained $\nu w=1095,1100$ and $1123 \mathrm{~m} / \mathrm{s}$ for samples NBP\#1,2 and 3 respectively. These values are close to the frequency of the sphere $(\nu d=1062 \mathrm{~m} / \mathrm{s})$ and about $10 \%$ larger than those of the cylinder.

Next we consider the well-known length-dependent extensional modes for the bipyramids. Such vibrations have already been measured using femtosecond pump-probe experiments and their frequencies were also determined analytically. ${ }^{46}$ Using the actual average sizes reported in Table 1, we calculated the extensional mode frequencies using this approximation and 
compared with finite element calculations.

The extensional modes calculated using the analytical solution and assuming flat ends are as follows:

$\mathrm{NBP} \# 1: \nu w=522,1320,2159,3006,3856, \ldots \mathrm{m} / \mathrm{s}$

NBP\#2: $\nu w=512,1263,2058,2862,3670,4479, \ldots \mathrm{m} / \mathrm{s}$

NBP\#3: $\nu w=525,1146,1813,2497,3187,3881,4577,5274,5972, \ldots \mathrm{m} / \mathrm{s}$

The extensional modes of pentagonal NBPs have the $A_{i}^{\prime}$ symmetry. The first $A_{i}^{\prime}$ modes are as follows:

$\mathrm{NBP} \# 1: \nu w=586,1353,1830,1947,2231, \ldots \mathrm{m} / \mathrm{s}$

$\mathrm{NBP} \# 2: \nu w=566,1304,1795,1975,2187,2324, \ldots \mathrm{m} / \mathrm{s}$

NBP\#3: $\nu w=550,1169,1685,2076,2095,2425, \ldots \mathrm{m} / \mathrm{s}$

Comparing with the analytical calculations confirms that only the lowest frequency ones are "pure" extensional modes. At higher frequency, diameter-dependent modes having the same symmetry also manifest.

Using the calculated frequencies reported in Figure $3 \mathrm{~A}$ and the ones calculated for the extensional vibrations, we propose the following assignment. NBP\#1 peaks at $\nu w=611$ and $1264 \mathrm{~m} / \mathrm{s}$ and NBP\#2 peaks at 660 and $1298 \mathrm{~m} / \mathrm{s}$ are assigned to the first 2 extensional modes. NBP\#1 peak at $\nu w=989 \mathrm{~m} / \mathrm{s}$ and NBP\#2 peak at $\nu w=1021 \mathrm{~m} / \mathrm{s}$ are assigned to the " $m=2$ " mode at $\nu w=999 \mathrm{~m} / \mathrm{s}$. The broad feature observed at $\nu w=1670 \mathrm{~m} / \mathrm{s}$ for NBP\#2 is close to the " $m=3$ " mode at $\nu w=1601 \mathrm{~m} / \mathrm{s}$. An additional narrow feature is observed for $\mathrm{NBP} \# 1$ at about $\nu w=775 \mathrm{~m} / \mathrm{s}$. It does not correspond to one of the vibrations described above. Its frequency is close to that of the $\mathrm{E}_{1}^{\prime}$ mode which is the first overtone of the bending mode as calculated with the finite element approach $(\nu w=747 \mathrm{~m} / \mathrm{s})$.

The peaks observed for NBP\#3 have significantly larger values of $\nu w$. Most of the modes observed for NBP\#1 and 2 are masked in the tail of the elastic peak for NBP\#3. To assign the peaks, we first look at the modes corresponding to larger values of " $m$ " as 
done before for the NRs. Peaks are observed at about $\nu w=1540,2260,3120,3820$ and $5030 \mathrm{~m} / \mathrm{s}$. The first value is close to the one for $\mathrm{E}_{2}^{\prime}(m=3)$. The following ones match approximately the following values for odd " $m$ " ( $\nu d=1452,2326,3138$ and $3928 \mathrm{~m} / \mathrm{s}$ for $m=3,5,7$ and 9$)$. This assignment is only tentative at this point as it requires a thorough investigation of a larger set of the full parameter space (length and diagonals at the center and at the ends) which goes beyond the scope of the present work. Still, we would like to emphasize that it is consistent with the deviations from the Raman selection rules because of the plasmonic coupling as described above in the case of NRs. First, we note that the size of $\mathrm{NBP} \# 3$ is almost twice that of $\mathrm{NBP} \# 1$ and $\mathrm{NBP} \# 2$. We can also assume that the distance $s$ between neighbor NBPs is similar for each sample because it is governed by the length of the ligands. The $s / D$ ratio is therefore much smaller in NBP\#3 compared to NBP\#1 and NBP\#2 resulting in a significantly larger plasmonic coupling. This probably explains why the assignment of the peaks for NBP\#1 and NBP\#2 did not require considering $m>2$ modes while such vibrations are involved for NBP\#3. In addition, the SEM and TEM images show that NBPs do not self-assemble as easily as NRs. After deposition, their arrangement looks mostly random. However, we can point out that NRs tend to self-assemble so that their ends are aligned while neighbor NBPs are most often shifted so that the tip of one NBP is close to the center of an adjacent NBP. These differences probably result in a very different symmetry of the electric field inside NBPs compared to NRs which may be the reason for the unexpected odd " $m$ " observation reported above.

\section{Conclusion}

Gold NRs and NBPs were synthesized. Their morphology was determined with electron microscopy and optical absorption. The nanocrystals are tens of nanometer large monodomain gold NRs and twinned pentagonal NBPs with narrow size distributions. Their vibrational dynamics was investigated experimentally by means of inelastic light scattering. Numerous 
peaks were observed. The vibrations were modeled in the framework of continuum elasticity. Special care was taken to identify their symmetry and compare them to the vibrations of nanowires as the limiting case of very high aspect ratio NRs. We proposed a method to assign the experimental peaks following recent results for gold nanodimers. ${ }^{[19}$ For NRs, the frequencies of the peaks match those of the fundamental spheroidal vibrations which couple with the surface plasmon resonance by changing the shape of the surface. Deviations from the usual low-frequency Raman selection rules are observed because they are derived for small and isolated nanoparticles. ${ }^{47}$ Vibrations with angular momenta larger than 2 are involved more and more as the ratio of the interparticle separation to the diameter decreases. The agreement between the experimental and calculated frequencies is good for NRs. The assignment is more complex for large NBPs for which different selection rules seem to be at work because of the different arrangement of the nanocrystals after deposition. However, the unusually large number of peaks observed for the largest NBPs, which occur at large values of the frequency $\times$ diagonal product, can not be explained without involving vibrations having larger angular momenta. These results show that inelastic light scattering in the $\mathrm{GHz}$ frequency range is a powerful probe of the plasmonic coupling in assemblies of nanocrystals. The assignment obtained in this work is general but intensity variations can be expected when varying the experimental parameters. Future works will focus on carefully monitoring the assembly of the nanocrystals being probed as well as polarization effects, resonance conditions and developing new methods to calculate the inelastic scattering cross-sections for non-spherical shapes.

\section{Methods}

\section{Syntheses}

Hydrogen tetrachloroaurate (Sigma-Aldrich, $\geq 99.9 \%$ ), silver nitrate (Sigma-Aldrich, 99\%), sodium borohydride (Sigma-Aldrich, $\geq 96 \%$ ), sodium hydroxide (VWR, $\geq 98 \%$ ), hydroquinone 
(Sigma-Aldrich, $\geq 99 \%$ ), cetyltrimethylammonium bromide (Sigma-Aldrich, $\geq 98 \%$ ), cetyltrimethylammonium chloride (Sigma-Aldrich, $\geq 99.5 \%$ ), benzyldimethylhexadecylammonium chloride (Sigma-Aldrich). All chemicals were used as received. All solutions were fresh daily prepared. All glassware were washed with aqua regia $\left(\mathrm{HNO}_{3} / \mathrm{HCl} 2: 1(\mathrm{v} / \mathrm{v})\right)$ and rinced with ultrapure water $(18.2 \mathrm{M} \Omega)$.

For gold NRs, the seed and growth solutions were prepared as follows.

- Seed solution: under strong stirring, $460 \mu \mathrm{L}$ of $\mathrm{NaBH}_{4}(0.01 \mathrm{M})$ and $\mathrm{NaOH}(0.01 \mathrm{M})$ solution was injected with one stroke in $10 \mathrm{~mL}$ of $\mathrm{HAuCl}_{4}(0.5 \mathrm{mM})$ and $\mathrm{CTAB}(0.1 \mathrm{M})$ solution at room temperature. The color turned from yellow-orange to brown.

- Growth solution: at $30^{\circ} \mathrm{C}$ and under slow stirring, 160, 240, 320, 400, 480, 560, 640, 720, 800 or $880 \mu \mathrm{L}$ of $\mathrm{AgNO}_{3}(0.01 \mathrm{M})$ solution was added to $40 \mathrm{~mL}$ of $\mathrm{HAuCl}_{4}(0.5 \mathrm{mM})$ and CTAB $(0.1 \mathrm{M})$ solution. $2 \mathrm{~mL}$ of hydroquinone was mixed to the growth solution. The color turned from yellow-orange to colorless. Finally, a volume of $640 \mathrm{~mL}$ of the seed solution was added and the growth solution was let undisturbed overnight.

Similarly, for gold NBPs, the seed and growth solutions were prepared as follows.

- Seed solution: under strong stirring, $100 \mu \mathrm{L}$ of $\mathrm{NaBH}_{4}(0.05 \mathrm{M})$ and $\mathrm{NaOH}(0.05 \mathrm{M})$ solution was injected with one stroke in $4 \mathrm{~mL}$ of $\mathrm{HAuCl}_{4}(0.5 \mathrm{mM})$ and CTAC $(95 \mathrm{mM})$ solution at room temperature. The color turned from yellow-orange to red. Ripening took place during one hour at $85^{\circ} \mathrm{C}$.

- Growth solution: at $30^{\circ} \mathrm{C}$ and under slow stirring, $0.8 \mathrm{~mL}$ of $\mathrm{AgNO}_{3}(0.01 \mathrm{M})$ solution was added to $40 \mathrm{~mL}$ of $\mathrm{HAuCl}_{4}(0.5 \mathrm{mM})$ and $\mathrm{CTAB}(0.1 \mathrm{M})$ solution. $2 \mathrm{~mL}$ of hydroquinone $(0.1 \mathrm{M})$ was mixed to the growth solution. The color turned from yelloworange to colorless. Finally, 1500, 1300, 1100, 900, 700, 500, 300, 150, 100 or $50 \mathrm{\mu L}$ of the seed solution was added and the growth solution was let undisturbed overnight.

For the purification of the nanocrystals, $10 \mathrm{~mL}$ of solution was centrifuged during $15 \mathrm{~min}$ at $4500 \mathrm{rpm}$ and $40^{\circ} \mathrm{C}$. The precipitate was redipersed in $10 \mathrm{~mL}$ of CTAB solution $(1 \mathrm{mM})$. 
Centrifugation was repeated one more time and the precipitated nanocrystals were redispersed in $3 \mathrm{~mL}$ of CTAB solution $(1 \mathrm{mM})$. The nanocrystals were redispersed with BDAC solution $(0.5 \mathrm{M})$ to a total concentration: $[\mathrm{BDAC}]=651.52-4.41 L$ with $L$ the nanocrystal length. After precipitation during $18 \mathrm{~h}$, gold nanocrystals were redispersed in $3 \mathrm{~mL}$ of CTAB solution $(1 \mathrm{mM})$. The solution was centrifuged during $5 \mathrm{~min}$ at $5000 \mathrm{rpm}$ and $40^{\circ} \mathrm{C}$. The precipitate was redipersed in $3 \mathrm{~mL}$ of CTAB solution $(1 \mathrm{mM})$. Centrifugation was repeated one more time and the precipitated nanocrystals were redispersed in $3 \mathrm{~mL}$ of CTAB solution $(1 \mathrm{mM})$.

\section{Material characterization}

UV visible absorption spectra of the colloidal solutions were recorded using a Varian 50 scan spectrophotometer. For conventional TEM and HRTEM, we used a JEOL 1011, JEOL 2010 and 2010F, respectively. The samples were prepared by depositing a drop of colloidal solution onto a copper grid covered by an amorphous carbon film. The size distributions were determined using the NIH ImageJ software. HRSEM pictures were acquired with a HITACHI SU-70 instrument. The low-frequency Raman scattering measurements were performed using a tandem Fabry-Pérot interferometer. The scattered light was collected in the backscattering configuration. The excitation source was the $647.1 \mathrm{~nm}$ line of a $\mathrm{Kr}^{+}$laser. For Raman measurements, either Au NRs or NBPs were deposited onto a silicon wafer by evaporation of the colloidal solution in a small beaker.

\section{Calculation of the acoustic eigenmodes}

The calculation of the eigenvibrations was performed using the Rayleigh-Ritz variational method as explained in a previous work by one of us. $\frac{39}{39}$ The point group for single-domain NRs having their main axis along [001] is $\mathrm{D}_{4 \mathrm{~h}}$ and the one for the pentagonal bipyramids is $\mathrm{D}_{5 \mathrm{~h}}$. The intensity of inelastically scattered light is also calculated by considering a Brillouin mechanism, i.e., a constant electric field inside the nanocrystals. This approximation is 
poor ${ }^{39}$ but we use it as a rough guide since it is easy enough to calculate, and we are lacking a better model.

In the case of the NBPs, numerical issues resulted in a poor convergence using the previous approach. It was therefore replaced by finite element calculations (Abaqus/CAE 6.18). We used the cyclic analysis method which takes advantage of the 5-fold symmetry axis $\left(\mathrm{C}_{5}\right)$ and provides the nodal diameter for each eigenvibration. Additional characters (symmetry about the plane perpendicular to $\mathrm{C}_{5}$ and one of the 5 planes containing $\mathrm{C}_{5}$ ) were evaluated numerically to complete the determination of the irreducible representations.

\section{Acknowledgement}

The authors acknowledge IMPC (Institut des Matériaux de Paris Centre, FR2482) for providing access to the JEOL1011 and JEOL2010 microscopes. N.G. acknowledges the support of Nouraine Fadili for the synthesis of gold nanorods. This work has been supported by the EIPHI Graduate School (contract "ANR-17-EURE-0002"). Calculations were performed using HPC resources from DNUM CCUB (Centre de Calcul de l'Université de Bourgogne).

\section{Supporting Information Available}

SEM and HRSEM images for NRs self-organized on a Si wafer, TEM image showing single domain NRs, and diameter and length distributions for samples NR\#1-6. HRTEM image of one single domain Au NR. Stokes and anti-Stokes low-frequency inelastic light scattering spectra of samples NR\#1-6. SEM and HRSEM images for NBPs self-organized on a Si wafer, TEM image showing multiply-twinned NBPs along with few nanoparticles of different shapes (spheroids, NRs), and diagonal and length distributions for samples NBP\#1-3. HRTEM image of one multiply-twinned Au NBP. Stokes and anti-Stokes low-frequency inelastic light scattering spectra of samples NBP\#1-3. Visible-NIR absorption spectra of colloidal solutions of samples NR\#1-6 and NBP\#1-3. Frames and animations of the radial vibrations of an 
isotropic Au nanowire with $m=2,3,4$ and 5 .

This material is available free of charge via the Internet at https://pubs.acs.org.

\section{References}

1. Murphy, C. J.; Sau, T. K.; Gole, A. M.; Orendorff, C. J.; Gao, J.; Gou, L.; Hunyadi, S. E.; Li, T. Anisotropic Metal Nanoparticles: Synthesis, Assembly, and Optical Applications. J. Phys. Chem. B 2005, 109, 13857-13870.

2. Mackey, M. A.; Ali, M. R. K.; Austin, L. A.; Near, R. D.; El-Sayed, M. A. The Most Effective Gold Nanorod Size for Plasmonic Photothermal Therapy: Theory and In Vitro Experiments. J. Phys. Chem. B 2014, 118, 1319-1326.

3. Amendola, V.; Pilot, R.; Frasconi, M.; Maragò, O. M.; Iatì, M. A. Surface Plasmon Resonance in Gold Nanoparticles: A Review. J. Phys.: Condens. Matter 2017, 29, 203002.

4. Lohse, S. E.; Murphy, C. J. The Quest for Shape Control: A History of Gold Nanorod Synthesis. Chem. Mater. 2013, 25, 1250-1261.

5. Weitz, D. A.; Gramila, T. J.; Genack, A. Z.; Gersten, J. I. Anomalous Low-Frequency Raman Scattering from Rough Metal Surfaces and the Origin of Surface-Enhanced Raman Scattering. Phys. Rev. Lett. 1980, 45, 355-358.

6. Montagna, M.; Dusi, R. Raman Scattering from Small Spherical Particles. Phys. Rev. B 1995, 52, 10080-10089.

7. Hodak, J. H.; Martini, I.; Hartland, G. V. Observation of Acoustic Quantum Beats in Nanometer Sized Au Particles. J. Chem. Phys. 1998, 108, 9210-9213.

8. Hamanaka, Y.; Kuwabata, J.; Tanahashi, I.; Omi, S.; Nakamura, A. Ultrafast Electron 
Relaxation via Breathing Vibration of Gold Nanocrystals Embedded in a Dielectric Medium. Phys. Rev. B 2001, 63, 104302.

9. Bachelier, G.; Mlayah, A. Surface Plasmon Mediated Raman Scattering in Metal Nanoparticles. Phys. Rev. B 2004, 69, 205408.

10. Mazurenko, D. A.; Shan, X.; Stiefelhagen, J. C. P.; Graf, C. M.; van Blaaderen, A.; Dijkhuis, J. I. Coherent Vibrations of Submicron Spherical Gold Shells in a Photonic Crystal. Phys. Rev. B 2007, 75, 161102.

11. Portalès, H.; Goubet, N.; Saviot, L.; Adichtchev, S.; Murray, D. B.; Mermet, A.; Duval, E.; Pileni, M.-P. Probing Atomic Ordering and Multiple Twinning in Metal Nanocrystals through their Vibrations. Proc. Natl. Acad. Sci. U. S. A. 2008, 105, 1478414789.

12. Pelton, M.; Sader, J. E.; Burgin, J.; Liu, M.; Guyot-Sionnest, P.; Gosztola, D. Damping of Acoustic Vibrations in Gold Nanoparticles. Nat. Nanotechnol. 2009, 4, 492-495.

13. Jais, P. M.; Murray, D. B.; Merlin, R.; Bragas, A. V. Metal Nanoparticle Ensembles: Tunable Laser Pulses Distinguish Monomer from Dimer Vibrations. Nano Lett. 2011, $11,3685-3689$.

14. Dacosta Fernandes, B.; Spuch-Calvar, M.; Baida, H.; Tréguer-Delapierre, M.; Oberlé, J.; Langot, P.; Burgin, J. Acoustic Vibrations of Au Nano-Bipyramids and their Modification under Ag Deposition: A Perspective for the Development of Nanobalances. ACS Nano 2013, 7, 7630-7639.

15. Yu, K.; Zijlstra, P.; Sader, J. E.; Xu, Q.-H.; Orrit, M. Damping of Acoustic Vibrations of Immobilized Single Gold Nanorods in Different Environments. Nano Lett. 2013, 13, 2710-2716. 
16. Major, T. A.; Crut, A.; Gao, B.; Lo, S. S.; Fatti, N. D.; Vallée, F.; Hartland, G. V. Damping of the Acoustic Vibrations of a Suspended Gold Nanowire in Air and Water Environments. Phys. Chem. Chem. Phys. 2013, 15, 4169-4176.

17. Bayle, M.; Combe, N.; Sangeetha, N. M.; Viau, G.; Carles, R. Vibrational and Electronic Excitations in Gold Nanocrystals. Nanoscale 2014, 6, 9157-9165.

18. Kirschner, M. S.; Lethiec, C. M.; Lin, X.-M.; Schatz, G. C.; Chen, L. X.; Schaller, R. D. Size-Dependent Coherent-Phonon Plasmon Modulation and Deformation Characterization in Gold Bipyramids and Nanojavelins. ACS Photonics 2016, 3, 758-763.

19. Girard, A.; Lermé, J.; Gehan, H.; Mermet, A.; Bonnet, C.; Cottancin, E.; Crut, A.; Margueritat, J. Inelastic Light Scattering by Multiple Vibrational Modes in Individual Gold Nanodimers. J. Phys. Chem. C 2019, 123, 14834-14841.

20. Valley, D. T.; Ferry, V. E.; Flannigan, D. J. Imaging Intra- and Interparticle AcoustoPlasmonic Vibrational Dynamics with Ultrafast Electron Microscopy. Nano Lett. 2016, $16,7302-7308$.

21. Kim, Y.-J.; Jung, H.; Han, S. W.; Kwon, O.-H. Ultrafast Electron Microscopy Visualizes Acoustic Vibrations of Plasmonic Nanorods at the Interfaces. Matter 2019, 1, 481-495.

22. Pelton, M.; Chakraborty, D.; Malachosky, E.; Guyot-Sionnest, P.; Sader, J. E. Viscoelastic Flows in Simple Liquids Generated by Vibrating Nanostructures. Phys. Rev. Lett. 2013, 111, 244502 .

23. Girard, A.; Lermé, J.; Gehan, H.; Margueritat, J.; Mermet, A. Mechanisms of Resonant Low Frequency Raman Scattering from Metallic Nanoparticle Lamb Modes. J. Chem. Phys. 2017, 146, 194201.

24. Leite, R. C. C.; Scott, J. F.; Damen, T. C. Multiple-Phonon Resonant Raman Scattering in CdS. Phys. Rev. Lett. 1969, 22, 780-782. 
25. Dresselhaus, M.; Dresselhaus, G.; Saito, R.; Jorio, A. Raman Spectroscopy of Carbon Nanotubes. Phys. Rep. 2005, 409, 47-99.

26. Ferrari, A. C.; Meyer, J. C.; Scardaci, V.; Casiraghi, C.; Lazzeri, M.; Mauri, F.; Piscanec, S.; Jiang, D.; Novoselov, K. S.; Roth, S.; Geim, A. K. Raman Spectrum of Graphene and Graphene Layers. Phys. Rev. Lett. 2006, 97, 187401.

27. Fujii, M.; Nagareda, T.; Hayashi, S.; Yamamoto, K. Low-Frequency Raman Scattering from Small Silver Particles Embedded in $\mathrm{SiO}_{2}$ Thin Films. Phys. Rev. B 1991, 44, 6243-6248.

28. Fujii, M.; Nagareda, T.; Hayashi, S.; Yamamoto, K. Erratum: Low-Frequency Raman Scattering from Small Silver Particles Embedded in $\mathrm{SiO}_{2}$ Thin Films. Phys. Rev. B 1995, 52, 14273-14273.

29. Beane, G.; Devkota, T.; Brown, B. S.; Hartland, G. V. Ultrafast Measurements of the Dynamics of Single Nanostructures: A Review. Rep. Prog. Phys. 2018, 82, 016401.

30. Ahmed, A.; Pelton, M.; Guest, J. R. Understanding How Acoustic Vibrations Modulate the Optical Response of Plasmonic Metal Nanoparticles. ACS Nano 2017, 11, 93609369.

31. Montagna, M. Brillouin and Raman Scattering from the Acoustic Vibrations of Spherical Particles with a Size Comparable to the Wavelength of the Light. Phys. Rev. B 2008, $77,045418$.

32. Kirschner, M. S.; Lethiec, C. M.; Lin, X.-M.; Schatz, G. C.; Chen, L. X.; Schaller, R. D. Size-Dependent Coherent-Phonon Plasmon Modulation and Deformation Characterization in Gold Bipyramids and Nanojavelins. ACS Photonics 2016, 3, 758-763.

33. Large, N.; Saviot, L.; Margueritat, J.; Gonzalo, J.; Afonso, C. N.; Arbouet, A.; Lan- 
got, P.; Mlayah, A.; Aizpurua, J. Acousto-Plasmonic Hot Spots in Metallic NanoObjects. Nano Lett. 2009, 9, 3732-3738.

34. Miscuglio, M.; Lin, M.; Di Stasio, F.; Tan, P.-H.; Krahne, R. Confined Acoustic Phonons in Colloidal Nanorod Heterostructures Investigated by Nonresonant Raman Spectroscopy and Finite Elements Simulations. Nano Lett. 2016, 16, 7664-7670.

35. Gan, Y.; Sun, Z.; Chen, Z. Breathing Mode Vibrations and Elastic Properties of SingleCrystal and Penta-Twinned Gold Nanorods. Phys. Chem. Chem. Phys. 2016, 18, 2259022598 .

36. Goupalov, S. V. Crystal Structure Anisotropy Explains Anomalous Elastic Properties of Nanorods. Nano Lett. 2014, 14, 1590-1595.

37. Goupalov, S. V. Correction to "Crystal Structure Anisotropy Explains Anomalous Elastic Properties of Nanorods". Nano Lett. 2014, 14, 2232-2232.

38. Burgin, J.; Langot, P.; Arbouet, A.; Margueritat, J.; Gonzalo, J.; Afonso, C. N.; Vallée, F.; Mlayah, A.; Rossell, M. D.; Tendeloo, G. V. Acoustic Vibration Modes and Electron-Lattice Coupling in Self-Assembled Silver Nanocolumns. Nano Lett. 2008, 8, $1296-1302$.

39. Saviot, L. Vibrations of Single-Crystal Gold Nanorods and Nanowires. Phys. Rev. B 2018, 97, 155420 .

40. Liu, M.; Guyot-Sionnest, P. Mechanism of Silver(I)-Assisted Growth of Gold Nanorods and Bipyramids. J. Phys. Chem. B 2005, 109, 22192-22200.

41. Saviot, L.; Murray, D. B. Longitudinal versus Transverse Spheroidal Vibrational Modes of an Elastic Sphere. Phys. Rev. B 2005, 72, 205433.

42. Margueritat, J.; Gonzalo, J.; Afonso, C. N.; Mlayah, A.; Murray, D. B.; Saviot, L. 
Surface Plasmons and Vibrations of Self-Assembled Silver Nanocolumns. Nano Lett. 2006, 6, 2037-2042.

43. Portalès, H.; Goubet, N.; Saviot, L.; Yang, P.; Sirotkin, S.; Duval, E.; Mermet, A.; Pileni, M.-P. Crystallinity Dependence of the Plasmon Resonant Raman Scattering by Anisotropic Gold Nanocrystals. ACS Nano 2010, 4, 3489-3497.

44. Vial, S.; Pastoriza-Santos, I.; Pérez-Juste, J.; Liz-Marzán, L. M. Plasmon Coupling in Layer-by-Layer Assembled Gold Nanorod Films. Langmuir 2007, 23, 4606-4611.

45. Jain, P. K.; Huang, W.; El-Sayed, M. A. On the Universal Scaling Behavior of the Distance Decay of Plasmon Coupling in Metal Nanoparticle Pairs: A Plasmon Ruler Equation. Nano Lett. 2007, 7, 2080-2088.

46. Pelton, M.; Wang, Y.; Gosztola, D.; Sader, J. E. Mechanical Damping of Longitudinal Acoustic Oscillations of Metal Nanoparticles in Solution. J. Phys. Chem. C 2011, 115, $23732-23740$.

47. Duval, E. Far-Infrared and Raman Vibrational Transitions of a Solid Sphere: Selection Rules. Phys. Rev. B 1992, 46, 5795-5797. 


\section{Graphical TOC Entry}

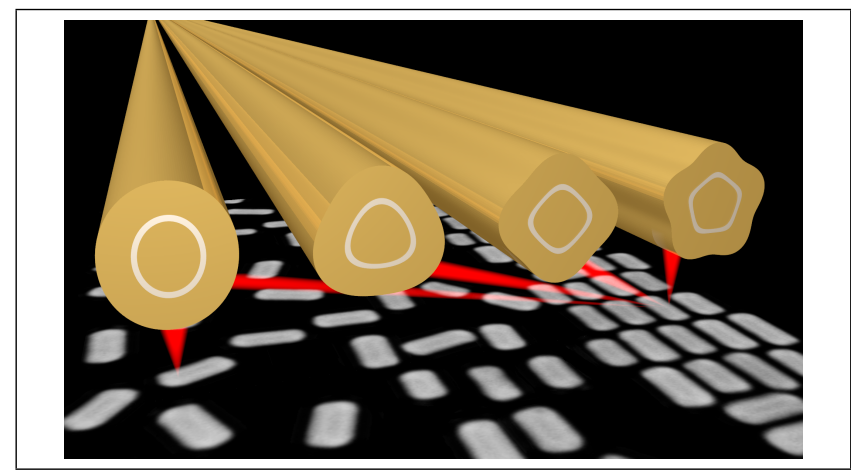

Raman scattering from $m>2$ spheroidal vibrations is observed when gold nanorods are very close. 